Pure and Applied Mathematics Quarterly

Volume 7, Number 3

(Special Issue: In honor of

Jacques Tits)

$1037-1052,2011$

\title{
Free Polygons, Twin Trees, and CAT(1)-Spaces
}

\author{
Katrin Tent
}

\begin{abstract}
We give model theoretic constructions for a zoo of examples and counterexamples: first we build generalized $n$-gons satisfying strong transitivity properties. An ultraproduct of these yields a forest from which we obtain twin trees as well as homogeneous 1-round CAT(1)-spaces.
\end{abstract}

Keywords: generalized polygons, twin trees, CAT(1)-spaces

\section{Introduction}

The classification of the Moufang polygons by Tits and Weiss [18] establishes a close connection between Moufang polygons (and higher rank spherical buildings) and classical or algebraic groups. Weak versions of the Moufang condition have been shown to be sufficient for the classification [13, 14, 15]. While in the finite case and in the case of compact connected topological buildings the Moufang condition is in fact equivalent to the existence of a BN-pair, examples (such as the ones constructed below) show that this fails in general. Therefore, it is of interest to explore the extend to which the Moufang condition might be weakened. We here construct examples satisfying rather strong homogeneity conditions. This construction is much more straightforward than the one given in [12] and has different properties. In Section 5 we use these examples to obtain twin trees with

Received March 9, 2009.

MSC 20E42,51E12, 05C25 
large automorphism groups. Finally we consider the ultralimit of these spaces in Section 6.

\section{Generalized polygons - free constructions}

Generalized polygons are exactly the spherical buildings of rank 2. A generalized $n$-gon $\Gamma$ is a bipartite graph with valencies at least 3 , diameter $n$ and girth $2 n$. Without the assumption on the valencies, such a graph is called a weak $\mathrm{n} n$-gon. We call $\left(x_{0}, \ldots x_{k}\right)$ a simple path if the $x_{i}$ are pairwise distinct and $x_{i}$ is adjacent to $x_{i+1}$ for $i=0, \ldots k-1$. The natural graph theoretic distance function on $\Gamma$ is denoted by $d$ or sometimes $d_{n}$. The set of elements at distance $i$ from some element $x \in \Gamma$ is denoted by $\Gamma_{i}(x)$. Elements at distance $n$ are called opposite.

The following easy lemma is a special case of [8].

2.1 Lemma If $\Gamma$ is a generalized $n$-gon and $\alpha \in \operatorname{Aut}(\Gamma)$, there exists some $x \in \Gamma$ with $d(x, \alpha(x)) \geq n-1$.

Proof. Let $x \in \Gamma$ be such that $k=d(x, \alpha(x))$ is maximal and suppose $k \leq$ $n-2$. For $i=1,2,3$ let $y_{i} \in \Gamma_{1}(x)$ and let $y_{1}$ be the unique element with $d\left(y_{1}, \alpha(x)\right)=k-1$. Clearly, $\alpha\left(y_{i}\right) \in \Gamma_{1}(\alpha(x))$. Therefore, there is at most one $i \in\{1,2,3\}$ with $d\left(y_{1}, \alpha\left(y_{i}\right)\right)=k-2$. Hence without loss of generality we have $d\left(y_{1}, \alpha\left(y_{1}\right)\right)=d\left(y_{1}, \alpha\left(y_{2}\right)\right)=k$ and therefore $d\left(y_{2}, \alpha\left(y_{2}\right)\right)=k+2$.

If $G \leq \operatorname{Aut}(\Gamma)$, we denote by $G_{x_{0}}^{[i]}$ the subgroup of $G$ fixing all elements of $\Gamma_{i}\left(x_{0}\right)$ and for elements $x_{0}, \ldots, x_{k}$, we set $G_{x_{0}, x_{1}, \ldots, x_{k}}^{[i]}=G_{x_{0}}^{[i]} \cap G_{x_{1}}^{[i]} \cap \ldots \cap G_{x_{k}}^{[1]}$.

For every simple path $\left(x_{0}, \ldots x_{n+1}\right)$ of length $n+1$ and every $i$ with $0 \leq i \leq n$, we have $G_{x_{0}, \ldots x_{n+1}} \cap G_{x_{i}, x_{i+1}}^{[1]}=1$ (see e.g. [20] $4.4 .2(\mathrm{v})$ ).

For $2 \leq k \leq n$, the generalized $n$-gon $\Gamma$ is said to be $k$-Moufang with respect to $G \leq \operatorname{Aut}(\Gamma)$ if for each simple $k$-path $\left(x_{0}, \ldots x_{k}\right)$ the group $G_{x_{1}, \ldots, x_{k-1}}^{[1]}$ acts transitively on the set of $2 n$-cycles through $\left(x_{0}, \ldots x_{k}\right)$. If $\Gamma$ is 4 -Moufang with respect to some group $G$, then $\Gamma$ is in fact $n$-Moufang with respect to the same group $G$ and we say that $\Gamma$ is a Moufang polygon (see e.g. [20] 6.8.2). If $G_{x_{0}, x_{1}}$ 
acts transitively on the set of $2 n$-cycles through $\left(x_{0}, x_{1}\right)$ for all paths $\left(x_{0}, x_{1}\right)$ (sometimes referred to as the 1-Moufang condition), then $G$ acts transitively on the set of ordered $2 n$-cycles of $\Gamma$, or strongly transitively on $\Gamma$. This is equivalent to $G$ having a spherical BN-pair of rank 2, which is in general too weak to allow a classification, see examples below and the ones in [17, 12].

We call a generalized $n$-gon $\Gamma$ almost-2-Moufang with respect to $G$ if for every finite set $A \subseteq \Gamma_{1}\left(x_{1}\right)$, and any path $\left(x_{0}, x_{1}, x_{2}\right)$ the group $G_{A}$ acts transitively on the $2 n$-cycles containing $\left(x_{0}, x_{1}, x_{2}\right)$. Similarly, one can define almost-3-Moufang for paths $\left(x_{0}, x_{1}, x_{2}, x_{3}\right)$ and finite subsets $A \subseteq \Gamma_{1}\left(x_{1}\right) \cup \Gamma_{1}\left(x_{2}\right)$.

It was shown in [15] that the 2-Moufang condition implies the Moufang condition for generalized $n$-gons with $n \leq 6$. We here construct generalized $n$-gons for all $n \geq 3$ which are almost-2-Moufang, but not Moufang (and in fact not even almost-3-Moufang)

It is well-known that finite or Moufang generalized $n$-gons exist only for $n=$ $3,4,6,8$. Background on the Moufang condition for generalized $n$-gons can be found in [20] and [18].

The following well-known construction shows the existence of many generalized $n$-gons for any $n \geq 3$.

2.2 Free $n$-completion: Let $\Gamma_{0}$ be a connected bipartite graph not containing any $k$-cycles for $k<2 n$. Then we obtain the free $n$-completion of $\Gamma_{0}$ in stages in the following way: at stage $i \geq 1$ we obtain $\Gamma_{i}$ from $\Gamma_{i-1}$ by adding a new path of length $n-1$ for each pair of elements $x, y$ at distance $n+1$ in $\Gamma_{i-1}$. Then $\Gamma=\bigcup \Gamma_{i}$ is called the free $n$-completion of $\Gamma_{0}$ and we say that $\Gamma$ is freely generated over $\Gamma_{0}$. If $\Gamma_{0}$ contains at least two pairs $x_{1}, y_{1}$ and $x_{2}, y_{2}$ of elements with $d\left(x_{1}, y_{1}\right)=d\left(x_{2}, y_{2}\right)=n+1$ in $\Gamma_{0}$ and $d\left(x_{1}, x_{2}\right)$ is prime to $n$, then $\Gamma$ is in fact a generalized $n$-gon (see e.g. [20] 1.3.13).

Obviously, if $\Gamma_{0} \cong \Delta_{0}$ then also their free $n$-completions are isomorphic, but the converse need not hold: the free $n$-completions of $\Gamma_{0}$ and $\Gamma_{i}$ are obviously the same.

However, there is a necessary criterion for the free completions of connected 
bipartite graphs to be isomorphic, which can be stated in terms of the rank function $\delta_{n}$.

2.3 Definition (i) For any finite graph $\Gamma=(V, E)$ with vertex set $V$ and edge set $E$ we define $\delta_{n}(\Gamma)=(n-1)|V|-(n-2)|E|$.

(ii) We say that the finite graph $\Gamma_{0}$ is $n$-strong in some graph $\Gamma$ (and we write $\left.\Gamma_{0} \leq_{n} \Gamma\right)$ if $\Gamma_{0} \subseteq \Gamma$ and for each finite graph $A \subseteq \Gamma, \Gamma_{0} \subseteq A$ we have $\delta_{n}\left(\Gamma_{0}\right) \leq \delta_{n}(A)$.

The function $\delta_{n}$ was denoted $y$ in [12] and was also used for $n=3$ in [5]. The $\leq$-relation was also considered in [12]. We will use the following easy fact (see [12] 2.4):

2.4 Fact If $A \leq_{n} B$ and $C \subseteq B$, then $A \cap C \leq_{n} C$.

We say that a generalized $n$-gon $\Gamma$ is generated by a subset $A \subseteq \Gamma$ if no generalized $n$-gon properly contained in $\Gamma$ contains $A . \Gamma$ is said to be finitely generated if it is generated by a finite subset. Similarly, a subpolygon $\Gamma^{\prime} \subseteq \Gamma$ is generated by $A \subseteq \Gamma$ if $\Gamma^{\prime}$ is the smallest subpolygon of $\Gamma$ containing $A$.

2.5 Proposition The following are equivalent for a generalized $n$-gon $\Gamma$ generated by a finite connected set $\Gamma_{0}$ :

(i) $\Gamma$ is the free $n$-completion of $\Gamma_{0}$;

(ii) $\Gamma_{0} \leq_{n} \Gamma$.

Proof. To see that (i) implies (ii), just notice that it follows immediately from the definition of $\delta_{n}$ and the definition of free extensions that we have $\delta_{n}\left(\Gamma_{0}\right)=\delta_{n}\left(\Gamma_{i}\right)$ for all $i$. Thus $\Gamma_{0} \leq_{n} \Gamma_{i}$ for all $i$. As any finite set $B$ containing $\Gamma_{0}$ will be contained in some stage $\Gamma_{i}$, the claim follows from 2.4.

For the other direction, suppose that $\Gamma_{0} \leq \Gamma$. We want to show that $\Gamma$ is the free completion of $\Gamma_{0}$. Let $\Gamma_{i}$ be the $i^{\text {th }}$ stage of the free completion of $\Gamma_{0}$. We 
show by induction on $i$ that $\Gamma_{i} \subseteq \Gamma$. The claim obviously holds for $i=0$. Now suppose $\Gamma_{i} \subseteq \Gamma$ and $x, y \in \Gamma_{i}$ have distance $n+1$ in $\Gamma_{i}$. Since $\Gamma$ is a generalized $n$-gon, there is a unique path $\left(x_{0}=x, x_{1}, \ldots x_{n-1}=y\right)$ in $\Gamma$. Clearly, this path cannot lie entirely in $\Gamma_{i}$. We claim that $x_{1}, \ldots x_{n-2} \notin \Gamma_{i}$. Suppose $x_{j} \in \Gamma_{i}$ with $1 \leq j \leq n-2$ minimal. Then $\Gamma_{i} \cup\left\{x_{1}, \ldots, x_{j-1}\right\}$ is a finite subgraph of $\Gamma$ containing $\Gamma_{0}$, but $\delta_{n}\left(\Gamma_{0}\right)=\delta_{n}\left(\Gamma_{i}\right)<\delta_{n}\left(\Gamma_{i} \cup\left\{x_{1}, \ldots, x_{j-1}\right\}\right)$ contradicting our assumption.

Since $\Gamma$ is generated by $\Gamma_{0}$ and $\bigcup \Gamma_{i}$ is a generalized polygon containing $\Gamma_{0}$, the claim follows.

2.6 Corollary Suppose $A_{0}$ and $B_{0}$ are finite connected graphs whose free $n$-completions $A$ and $B$ are isomorphic generalized n-gons. Then $\delta_{n}\left(A_{0}\right)=\delta_{n}\left(B_{0}\right)$.

Proof. Let $A_{0}, B_{0}$ be finite connected graphs and let $A, B$ be their respective $n$-completions. Suppose that $\delta_{n}\left(A_{0}\right)>\delta_{n}\left(B_{0}\right)$, but $A \cong B$ via some isomorphism $\varphi$. Then $\varphi\left(A_{0}\right)$ is contained in some finite stage $B_{i}$ of the free completion and $\varphi^{-1}\left(B_{i}\right)$ contains $A_{0}$. But clearly $\delta_{n}\left(\varphi^{-1}\left(B_{i}\right)\right)=\delta_{n}\left(B_{i}\right)=\delta_{n}\left(B_{0}\right)<\delta_{n}\left(A_{0}\right)$, contradicting Proposition 2.5.

2.7 Lemma If $\Gamma$ is a generalized $n$-gon freely generated over the finite set $\Gamma_{0}$, then every finitely generated sub-n-gon $\Delta$ is also freely generated over a finite set.

Proof. This is clear since there is some stage $\Gamma_{i}$ of the free $n$-completion which contains every element of the finite generating set for $\Delta$. Then $\Delta$ is the free completion of $\Delta \cap \Gamma_{i}$.

2.8 Corollary The class of generalized n-gons freely generated over a finite connected subgraph is countably infinite.

Proof. Each simple path $\gamma$ of length $k \geq n+2$ gives rise to a generalized $n$-gon. Notice that $\delta_{n}(\gamma)=(n-2)+k$ and so paths of different lengths give rise to nonisomorphic polygons. On the other hand, clearly, there are at most countably many finite connected graphs. 
2.9 Remark Note that by Tits' fundamental work [16], spherical buildings of rank greater than two all arise from algebraic groups. Thus, there is no way to obtain similar free constructions in this case.

\section{Amalgamation}

We will use Fraïssé's Amalgamation technique for a first order language to obtain new generalized polygons. Fraïssé's Theorem states the following (see [6] 7.1.2 for a proof):

3.1 Theorem Suppose $L$ is a first-order language and $\mathcal{C}$ is a class of finitely generated $L$-structures which is closed under finitely generated substructures satisfying the following additional properties:

- (Joint Embedding Property) for $A, B \in \mathcal{C}$, there is some $C \in \mathcal{C}$ such that both $A$ and $B$ are embeddable in $C$;

- (Amalgamation Property) for $A, B, C \in \mathcal{C}$, and embeddings $e: A \longrightarrow B, f$ : $A \longrightarrow C$, there is some $D \in \mathcal{C}$ and embeddings $g: B \longrightarrow D, h: C \longrightarrow D$ such that $g e=h f$

Then there is a countable L-structure $M$, unique up to isomorphism, satisfying:

(i) Every finitely generated substructure of $M$ is isomorphic to an element of $\mathcal{C}$;

(ii) Every element of $\mathcal{C}$ embeds into $M$.

(iii) If $A \in \mathcal{C}$, then $\operatorname{Aut}(M)$ acts transitively on the set of substructures of $M$ isomorphic to $A$.

The model $M$ is also called the Fraïssé limit of the class $\mathcal{C}$. Note that Fraïssé limits are existentially closed (that is, if there is some existential sentence which is true in some extension of the structure then it is already true in that structure). 
We now fix a first order language $L=\left\{f_{k}: k \in \mathbb{N}\right\}$ containing binary functions $f_{k}$. Any (partial) generalized $n$-gon becomes an $L$-structure if we interpret these functions as follows: $f_{k}(x, y)=x_{k}$ if $\left(x=x_{0}, \ldots, x_{k}, \ldots y\right)$ is the unique shortest path from $x$ to $y$. If there is no unique such path, then we let $f_{k}(x, y)=x$. Notice that edges of the graph can be defined in this language: in a generalized $n$-gon $\Gamma$ the pair $(x, y)$ is an edge if and only if $f_{1}(x, y)=y$. Thus, the axioms of a generalized $n$-gon are expressible in this language.

Clearly, the language $L$ has the following property: If $\Gamma$ is a generalized $n$-gon and $A \subseteq \Gamma$, then the $L$-substructure $\langle A\rangle$ of $\Gamma$ generated by $A$ is the same as the (possibly weak) sub- $n$-gon generated by $A$ in $\Gamma$.

3.2 Definition Let $\mathcal{C}_{n}$ be the class of all finitely generated $L$-substructures of all free $n$-completions of finite connected bipartite graphs not containing any $k$-cycles for $k<2 n$.

Then $\mathcal{C}_{n}$ is countable by Corollary 2.8 and closed under finitely generated $L$ substructures.

3.3 Remark $\mathcal{C}_{n}$ contains in particular the following structures:

(i) the empty structure (making the Joint Embedding Property a special case of the Amalgamation Property);

(ii) all paths of length at most $n$, and more generally any 'hat-rack', i.e. any path $\left(x_{0}, \ldots x_{k}\right), k \leq n$ together with finite subsets of $\Gamma_{1}\left(x_{i}\right), i=1, \ldots, k-1$;

(iii) a $2 n$-cycle, and more generally all finite weak $n$-gons containing at most 2 thick elements (at distance $n$ );

(iv) any finite generalized $n$-gon (these exist for $n=3,4,6,8$ only);

(v) arbitrarily large finite discrete sets if $n$ is even and a discrete set of order two if $n$ is odd.

In order to show that we may apply Fraïssé's Theorem to our class $\mathcal{C}_{n}$, it suffices to show that the Amalgamation Property holds for this class (as the empty structure 
is included in $\mathcal{C}_{n}$, which makes the Joint Embedding Property a special case of the Amalgamation Property.

3.4 Lemma $\mathcal{C}_{n}$ has the Amalgamation Property.

Proof. Let $A, B, C \in \mathcal{C}_{n}$, and suppose that $A$ is a substructure in both $B$ and $C$. Then let $D$ be the free $n$-completion of the free amalgam of $B$ and $C$ over $A$. Obviously, $D$ is in $\mathcal{C}_{n}$.

3.5 Theorem For all $n \geq 3$ there is a countable generalized $n$-gon $\Gamma_{n}$ whose automorphism group acts transitively on all finitely generated $L_{n}$-substructures of given isomorphism type.

Proof. This follows at once from Fraïssé's Theorem if we show that the Fraïssé limit $\Gamma_{n}$ of $\mathcal{C}_{n}$ is indeed a generalized $n$-gon. We first show that $\Gamma_{n}$ has diameter $n$. Let $x, y \in \Gamma_{n}$ and let $A$ be the $L_{n}$-substructure generated by $x, y$. Then either $A$ is a path from $x$ to $y$ (this happens if $d(x, y)<n$ ), or $A=\{x, y\}$. Let $\gamma=\left(x_{0}, \ldots x_{n}\right)$ be a path of length $n$, then $\gamma \in \mathcal{C}_{n}$ and hence $\gamma$ can be embedded into $\tilde{\Gamma}$. The images $z_{0}, z_{n}$ of $x_{0}, x_{n}$ will have distance $n$ in $\Gamma_{n}$, and hence $\left\{z_{0}, z_{n}\right\}$ is a finitely generated $L_{n}$-substructure of $\Gamma_{n}$ isomorphic to $A$. By homogeneity there is an automorphism of $\Gamma_{n}$ taking $\left\{z_{0}, z_{n}\right\}$ to $\{x, y\}$, taking a path from $z_{0}$ to $z_{n}$ to a path from $x$ to $y$ and proving $d(x, y)=n$ in $\Gamma_{n}$.

Clearly, $\Gamma_{n}$ does not contain any $k$-cycles for $k<2 n$ as such a cycle would generate a substructure isomorphic to an element of $\mathcal{C}_{n}$, which is impossible.

Finally, since the substructure generated by a single vertex in $\Gamma_{n}$ is just this vertex, the automorphism group is transitive on vertices. Now the existence of vertices of valency at least 3 immediately implies that all vertices have valency at least 3 .

3.6 Corollary In particular, $G=\operatorname{Aut}\left(\Gamma_{n}\right)$ has the following transitivity properties: 
(i) $G$ acts transitively on ordered $2 n$-cycles, so $G$ has a BN-pair;

(ii) for any $x \in \Gamma_{n}, G_{x}$ acts highly transitively on $\Gamma_{1}(x)$, i.e., $G_{x}$ is $k$-transitively for any $k \in \mathbb{N}$ on $\Gamma_{1}(x)$;

(iii) $G$ acts transitively on finite weak n-gons of the same cardinality. In particular, $\Gamma_{n}$ is almost-2-Moufang.

(iv) Let $\gamma=\left(x_{0}, x_{1}, \ldots x_{2 n}=x_{0}\right)$ be a $2 n$-cycle. Then the pointwise stabilizer of $\gamma$ acts highly transitively on $\Gamma_{1}\left(x_{1}\right) \backslash\left\{x_{0}, x_{2}\right\}$.

(v) For any finite set $A$ of vertices of $\Gamma$, the elements $\operatorname{Fix}\left(G_{A}\right)$ fixed by $G_{A}$ are exactly the substructure of $\Gamma_{n}$ generated by $A$.

(vi) $\Gamma_{n}$ is not almost-3-Moufang.

Proof. Properties (i)-(iv) all follow from the fact that the corresponding structures are isomorphic to elements of $\mathcal{C}_{n}$ and their isomorphism types are uniquely determined by their cardinality.

One inclusion of the claim in (v) is obvious. For the other inclusion, it suffices to notice that if $a \notin\langle A\rangle$, then there is $b \notin\langle A\rangle$ with $\langle A, a\rangle \cong\langle A, b\rangle$. Thus there is an automorphism of $\Gamma_{n}$ fixing $A$ pointwise and taking $a$ to $b$, so $a \notin \operatorname{Fix}\left(G_{A}\right)$.

For (vi), let $\gamma=\left(x_{0}, \ldots x_{2 n-1}, x_{0}\right)$ be a $2 n$-cycle and $a \in \Gamma_{1}\left(x_{1}\right) \backslash\left\{x_{0}, x_{2}\right\}, b \in$ $\Gamma_{1}\left(x_{2}\right) \backslash\left\{x_{1}, x_{3}\right\}$. Then the free $n$-completion $A$ of $\gamma \cup\{a, b\}$ and also a completion $B$ of $\gamma \cup\{a, b\}$ which is not free in a finite number of stages are both contained in $\mathcal{C}_{n}$. Since $G$ acts transitively on hat-racks of the form $\left(x_{0}, x_{1}, x_{2}, x_{3}\right) \cup\{a, b\}$, we find embeddings $\varphi_{1}, \varphi_{2}$ of $A$ and $B$ into $\Gamma$ which agree on $\left(x_{0}, x_{1}, x_{2}, x_{3}\right) \cup\{a, b\}$. The generalized polygons generated by the respective images of $\gamma \cup\{a, b\}$ in $\Gamma$ are not isomorphic, so there is no $g \in G_{a, b}$ taking $\phi_{1}(\gamma)$ to $\phi_{2}(\gamma)$.

3.7 Remark We have shown that for a path $\left(x_{0}, x_{1}, x_{2}, x_{3}\right)$ and $a \in \Gamma_{1}\left(x_{1}\right) \backslash$ $\left\{x_{0}, x_{2}\right\}$,

$b \in \Gamma_{1}\left(x_{2}\right) \backslash\left\{x_{1}, x_{3}\right\}$ the group $G_{a, b}$ is not transitive on $2 n$-cycles containing $\left(x_{0}, \ldots x_{3}\right)$. In particular, $\Gamma$ is not Moufang.

The proof of (vi) also shows that $G$ is not transitive on $2 n+2$-cycles of $\Gamma_{n}$ in contrast to the examples constructed in [12]. 


\section{Groups with highly transitive torus}

4.1 Definition Let $\Gamma$ be a generalized $n$-gon and $G \leq \operatorname{Aut}(\Gamma)$. Then $G$ has a highly transitive torus if for every $2 n$-cycle $\gamma=\left(x_{0}, x_{1}, \ldots x_{2 n}=x_{0}\right)$ the pointwise stabilizer $G_{\gamma}$ acts highly transitively on $\Gamma_{1}\left(x_{1}\right) \backslash\left\{x_{0}, x_{2}\right\}$.

Note that a highly transitive torus implies the existence of a BN-pair. Also, if $G$ has a highly transitive torus, then $G_{x}$ acts highly transitively on $\Gamma_{1}(x)$. As stated in Corollary 3.6, for each of the generalized $n$-gons $\Gamma_{n}$ constructed in Section 3, $\operatorname{Aut}\left(\Gamma_{n}\right)$ has a highly transitive torus.

We will need the following easy fact (see e.g. [4] Ex. 2.1.6):

4.2 Fact Every nontrivial normal subgroup of a highly transitive group is again highly transititive.

4.3 Lemma If $G$ has a highly transitive torus in its action on a generalized $n$-gon $\Gamma$, then for any $x \in \Gamma$, the group $G_{x}$ acts faithfully on $\Gamma_{1}(x)$.

Proof. Suppose $G_{x}^{[1]} \neq 1$. Let $i$ be maximal such that for some path $\left(x_{1} \ldots x_{i}\right)$ the group $G_{x_{1}, \ldots, x_{i}}^{[1]}$ is nontrivial. Then we must have $i<n$ and we can extend $\left(x_{1} \ldots x_{i}\right)$ to a $2 n$-cycle $\gamma=\left(x_{0}, x_{1}, \ldots, x_{2 n}=x_{0}\right)$. Let $\gamma^{\prime}=$ $\left(x_{j} \ldots x_{0}, x_{1} \ldots x_{i+1}, x_{i+2}, \ldots x_{k}\right)$ be a maximal path in $\gamma$ such that $H=G_{x_{1}, \ldots, x_{i}}^{[1]} \cap$ $G_{\gamma^{\prime}} \neq 1$. We claim that $\gamma^{\prime}=\gamma$. For if $H \neq 1$, then $H \unlhd G_{\gamma^{\prime}}$ acts highly transitively on $\Gamma_{1}\left(x_{k}\right) \backslash\left\{x_{k-1}\right\}$ and hence $H_{x_{k+1}} \neq 1$. Thus, $\gamma^{\prime}=\gamma$ and therefore $i=1$. Since $G_{x_{1}}^{[1]} \cap G_{\gamma}$ is normal in $G_{\gamma}$, it acts highly transitively on $\Gamma_{1}\left(x_{2}\right) \backslash\left\{x_{1}\right\}$. Let $u \in G_{x_{0}}^{[1]} \cap G_{x_{2}} \backslash G_{x_{3}}$. (Such an element exists.) Then for any $v \in\left(G_{x_{1}}^{[1]} \cap G_{\gamma}\right) \backslash G_{x_{3}^{u}}$ we have $[u, v] \in G_{x_{0}, x_{1}}^{[1]} \backslash\{1\}$, a contradiction.

4.4 Proposition Let $\Gamma$ be a generalized $n$-gon, and suppose that $G$ acts strongly transitively on $\Gamma$. Suppose that $\operatorname{Fix}\left(G_{x_{0}, x_{1}, x_{2}}\right)=\left\{x_{0}, x_{1}, x_{2}\right\}$ and $\operatorname{Fix}\left(G_{x_{0}, \ldots, x_{4}}\right)=$ $\left\{x_{0}, \ldots, x_{4}\right\}$ for any path $\left(x_{0}, x_{1}, x_{2}, x_{3}, x_{4}\right)$ of length 4 (where we allow $x_{1}=x_{3}$ ). Then $N_{x_{0}} \neq 1$ for any non-trivial normal subgroup $N$ of $G$. 
Proof. Since $G$ is primitive on vertices of a fixed type, $N$ is transitive on each type of vertices. If $N_{x_{0}}=1$, then $G=N \rtimes G_{x_{0}}$ and $N$ acts regularly on the vertices. Now let $y_{1}, y_{2} \in \Gamma_{2}\left(x_{0}\right)$ and for $i=1,2$ let $n_{i} \in N$ with $x_{0}^{n_{i}}=x_{i}$. Then $G_{x_{0}, y_{i}}$ equals the centralizer of $n_{i}$ in $G_{x_{0}}$. By assumption the only vertices of this type fixed by $G_{x_{0}, y_{i}}$ are $x_{0}$ and $y_{i}$ showing $n_{i}$ to be an involution for $i=1$, 2. Similarly we see that $n_{1} n_{2}$ is an involution. Thus, the elements of $N$ corresponding to $\Gamma_{2}\left(x_{0}\right)$ form an elementary abelian 2-group. This is invariant under the action of $G_{x_{0}}$ contradicting the maximality of $G_{x_{0}}$.

4.5 Theorem If $G$ has a highly transitive torus in its action on a generalized $n$-gon $\Gamma$, then any nontrivial normal subgroup $N$ also has a highly transitive torus and hence a BN-pair.

Proof. Let $\gamma=\left(x_{0}, x_{1}, \ldots x_{2 n}=x_{0}\right)$ be a $2 n$-cycle and let $N$ be a normal subgroup of $G$. By Lemma 4.2 it suffices to show that $N_{\gamma}$ is non-trivial.

Since $G$ has a highly transitive torus, it is easy to see that the conditions of Proposition 4.4 are satisfied and so $N_{x_{0}}$ is non-trivial and does not act trivially on $\Gamma_{1}\left(x_{0}\right)$ by Lemma 4.3. Since $N_{x_{0}} \unlhd G_{x_{0}}, N_{x_{0}}$ is highly transitive on $\Gamma_{1}\left(x_{0}\right)$. Thus, $N_{x_{0}, x_{1}}$ is nontrivial and acts nontrivially and hence highly transitively on $\Gamma_{1}\left(x_{1}\right)$ by Lemmas 4.3 and 4.2. Inductively, we thus show that $N_{\gamma}$ is nontrivial and acts nontrivially and hence highly transitively on $\Gamma_{1}\left(x_{1}\right)$, proving the claim.

The random graph can be constructed in an entirely similar way as the Fraisse limit of finite graphs. As its automorphism group turns out to be simple (see [19] or, more generally, [7]) we conjecture that also $G=\operatorname{Aut}\left(\Gamma_{n}\right)$ is simple. Theorem 4.5 seems a first step towards showing this simplicity.

\section{$5 \quad$ Twin trees and multiple trees}

Recall the definition of a twin tree [11]: 
5.1 Definition Let $T_{+}, T_{-}$be trees without endpoints. A twinning on $\left(T_{+}, T_{-}\right)$ is given by a codistance function $\operatorname{cod}: T_{+} \times T_{-} \cup T_{-} \times T_{+} \longrightarrow \mathbb{N}$ satisfying the following condition for all $x_{+} \in T_{+}, y_{-} \in T_{-}$:

If $\operatorname{cod}\left(x_{+}, y_{-}\right)=m$, then $\operatorname{cod}\left(x_{+}, y_{-}^{\prime}\right) \in\{m+1, m-1\}$ for every neighbour $y_{-}^{\prime}$ of $y_{-} \in T_{-}$. If $m>0$, there is a unique $y_{-}^{\prime}$ with $\operatorname{cod}\left(x_{+}, y_{-}^{\prime}\right)=m+1$, similarly with + and - interchanged.

We call $x_{+}$and $y_{-}$opposite if $\operatorname{cod}\left(x_{+}, y_{-}\right)=0$. Similarly, edges $e_{+}, e_{-}$are called opposite if the vertices of $e_{+}$are opposite the vertices of $e_{-}$. Recall also that a group $G$ acting transitively on the ordered doubly infinite paths through $T_{+}$has an affine BN-pair of type $\tilde{A}_{1}$. If $G$ acts codistance-preserving and transitively on pairs of opposite edges in $\left(T_{+}, T_{-}\right)$, then $G$ has a twin BN-pair of type $\tilde{A}_{1}$.

For each $n \in \mathbb{N}, n \geq 3$, we now fix a generalized almost 2-Moufang $n$-gon $\Gamma_{n}$ with a highly transitive torus as constructed in Section 3 and let $G_{n}=\operatorname{Aut}\left(\Gamma_{n}\right)$. We now consider the 3 -sorted structures consisting of the graphs $\Gamma_{n}$ with distance function $d$, its automorphism group $G_{n}$, and the natural numbers $\mathbb{N}$, where the distance function takes its values. Note that each $\Gamma_{n}$ comes with a natural opposition relation opp and codistance function cod defined by $\operatorname{cod}(x, y)=n-d(x, y)$ for $x, y \in \Gamma_{n}$. For $x, y \in \Gamma_{n}$ the codistance $\operatorname{cod}(x, y)$ is the smallest $k \leq n$ such that there is some $z$ opposite $x$ with $k=d(z, y)$.

Let $\mu$ be a nonprincipal ultrafilter on $\mathbb{N}$ and consider the ultraproduct

$$
\left(\Gamma, G, \mathbb{N}^{*}\right)=\Pi_{\mu}\left(\Gamma_{n}, G_{n}, \mathbb{N}\right)
$$

again in the language of metric graphs with a distance function $d$ taking values in $\mathbb{N}$. This ultraproduct is obtained from the cartesian product $\Pi_{n \in \mathbb{N}}\left(\Gamma_{n}, G_{n}, \mathbb{N}\right)$ by taking its elements to be equivalence classes modulo the equivalence relation $\sim_{\mu}$ where

$$
\left(a_{i}\right)_{i \in \mathbb{N}} \sim_{\mu}\left(b_{i}\right)_{i \in \mathbb{N}} \Leftrightarrow\left\{i \in \mathbb{N}: a_{i}=b_{i}\right\} \in \mu .
$$

We denote the elements of the ultraproduct by $\left(a_{n}\right)_{\mu}$. Then in the ultraproduct the sorts carry the structure of a graph with distance function $d$, a group of 
automorphisms of $\Gamma$ and a discretely ordered (non-archimedean) abelian semigroup, respectively. (See [6] for details.)

It is easy to see that $\Gamma$ is a forest, i.e., a graph with the property that each connected component is a tree, and the distance function takes values in $\mathbb{N}^{*}=$ $\Pi_{\mu} \mathbb{N}$. Let $\omega=(n)_{\mu} \in \mathbb{N}^{*}$, so $x, y \in \Gamma$ are opposite if and only if $d(x, y)=\omega$. Then $\Gamma$ is a generalized $\omega_{\mathbb{N}^{*}}$-gon in the sense of C. Bennett [2].

5.2 Theorem (i) If $T$ is a connected component of $\Gamma$, then $G_{\{T\}}$ acts faithfully on $T$ and has a BN-pair of type $\tilde{A}_{1}$. Here, $G_{\{A\}}$ denotes the setwise stabilizer of a set $A$.

(ii) Connected components $T_{1}, T_{2}$ of $\Gamma$ containing opposite vertices form a twin tree with the twinning given by $\operatorname{cod}(x, y)=\omega-d(x, y)$ for $x \in T_{1}, y \in T_{2}$.

(iii) If $T_{1}, T_{2}$ are connected components of $\Gamma$ containing opposite vertices, then $G_{\left\{T_{1}\right\}} \cap G_{\left\{T_{2}\right\}}$ has a twin BN-pair of type $\tilde{A}_{1}$.

Proof. Part (i) and (iii) follow from Lemma 4.3 and the fact that each $G_{n}$ acts transitively on pairs of opposite vertices in $\Gamma_{n}$. It is left to see that the condistance function cod : $T_{1} \times T_{2} \cup T_{2} \times T_{1} \longrightarrow \mathbb{N}^{*}$ has the required properties. Clearly, if $x \in T_{1}, y \in T_{2}$ are vertices with $\operatorname{cod}(x, y)=m \in \mathbb{N}^{*}$, then by the corresponding properties of each $\Gamma_{n}$ it is easy to see that if $m>0$, then $y$ has a unique neighbour $z \in T_{2}$ with $\operatorname{cod}(x, z)=m+1$ and $\operatorname{cod}\left(x, z^{\prime}\right)=m-1$ for all other neighbours $z^{\prime}$ of $y$. If $m=0$, then for all neighbours $z$ of $y$ the $\operatorname{codistance} \operatorname{cod}(z, x)=1$. Since $T_{1}, T_{2}$ contains a pair of opposite vertices, whose codistance is 0 , it follows inductively that for all $x \in T_{1}, y \in T_{2}$ we have in fact $\operatorname{cod}(x, y) \in \mathbb{N}$.

5.3 Remark $\bigcap G_{\{T\}}=1$ where the intersection is taken over all connected components $T$ of $\Gamma$ and if $T_{1}, T_{2}$ are connected components of $\Gamma$ containing opposite vertices, then $G_{T_{1} \cup T_{2}}=1$.

Proof. The first part follows easily from Lemma 2.1. The second part follows from the fact that $G_{x, y}^{[2]}=\{1\}$ for any group $G$ acting on a generalized $n$-gon with opposite elements $x, y$ (see e.g. [20] 4.4.2 (v)). 
If the even numbers have measure zero with respect to $\mu$ (i.e., $\{2 n: n \in \mathbb{N}\} \notin \mu$ ), then a set of pairwise opposite elements will have at most two elements, otherwise we can find a countably infinite such set by Remark 3.3. By pruning the trees adequately, it might also be possible to construct multiple trees in the sense of Ronan [10].

\section{Homogeneous CAT(1)-spaces}

For each $n \in \mathbb{N}, n \geq 3$, let $\Gamma_{n}$ and $G_{n}=\operatorname{Aut}\left(\Gamma_{n}\right)$ be as in Section 5 and let $\Gamma$ be the ultraproduct with respect to some nonprincipal ultrafilter $\mu$. We now construct the ultralimit $\tilde{\Gamma}$ of the (bounded metric) spaces $\Gamma_{n}$ from $\Gamma$ by scaling the $\mathbb{N}^{*}$-metric $d$ on $\Gamma$ by the factor $\pi / \omega$ and identifying points $x, y \in \Gamma$ such that $d(x, y) \pi / \omega<1 / n$ for all $n \in \mathbb{N}$. (Note that we could just as well have rescaled the metric on each $\Gamma_{n}$ by the factor $\pi / n$.) In particular, each connected component of $\Gamma$ is 'shrunk' to a point. By construction, $\tilde{\Gamma}$ contains antipodal points and any two antipodal points are contained in an isometrically embedded 1-sphere, but $\tilde{\Gamma}$ does not contain any manifold points. Similar examples without group actions were constructed by Nagano [9]. By Lemma 5.3 the group $G$ acts faithfully on $\tilde{\Gamma}$, and if $T$ is a connected component of $\Gamma$, then $G_{\{T\}} \leq G_{x}$ for some (any) point $x \in T$. Also, by construction $G$ acts transitively on the set of pointed 1-spheres contained in $\tilde{\Gamma}$.

Balser and Lytchak [1] prove that if $X$ is an $n$-dimensional CAT(1) space that has at least one pair of antipodes and such that each pair of antipodes is contained in some $S^{n}$, then $X$ is a spherical building provided that $X$ contains a relatively compact open subset. If a group acts transitively on the pointed $n$-spheres of a compact spherical building $X$, the work of Burns and Spatzier [3] shows that such a group is a non-compact real Lie group. Thus a similar statement is far from true without further topological assumptions.

\section{References}

[1] A. Balser, A. Lytchak, Building-like spaces. J. Math. Kyoto Univ. 46 (2006), no. 4, 789-804. 
[2] C. Bennett, Twin trees and $\lambda_{\Lambda}$-gons. Trans. Am. Math. Soc. 349, No.5, 2069-2084 (1997).

[3] K. Burns, R. Spatzier, On topological Tits buildings and their classification. Inst. Hautes Études Sci. Publ. Math. No. 65 (1987), 5-34.

[4] J. Dixon, B. Mortimer, Permutation groups. Graduate Texts in Mathematics. 163. New York, NY: Springer-Verlag (1996).

[5] M. Hall, Projective planes. Trans. Amer. Math. Soc. 54, (1943). 229 277.

[6] W. Hodges, A shorter model theory, Cambridge University Press, 1997.

[7] D. Macpherson, K. Tent, Simplicity of certain automorphism groups, preprint.

[8] B. Kleiner, B. Leeb, Rigidity of quasi-isometries for symmetric spaces and Euclidean buildings. Inst. Hautes Études Sci. Publ. Math. No. 86 (1997), 115-197 (1998).

[9] K. Nagano, Free construction of $C A T(1)$-spaces, Arch. Math. (Basel) 88 (2007), no. $2,173-180$.

[10] M. Ronan, Multiple trees. J. Algebra 271 (2004), no. 2, 673-697.

[11] M. Ronan, J. Tits, Twin trees. I. Invent. Math. 116 (1994), no. 1-3, 463-479.

[12] K. Tent, Very homogeneous generalized $n$-gons of finite Morley rank. J. Lond. Math. Soc., II. Ser. 62, No.1, 1-15 (2000).

[13] K. Tent, Half-Moufang implies Moufang for generalized quadrangles; in: J. Reine Angew. Math. 566 (2004).

[14] K. Tent, Half-Moufang generalized hexagons, Israel Journal of Mathematics 141 (2004), 83-92.

[15] K. Tent, A weak Moufang condition suffices. Eur. J. Comb. 26, No. 8, 1207-1215 (2005). 
[16] J. Tits, Buildings of spherical type and finite BN-pairs, Springer Lecture Notes in Mathematics, Vol. 386, 1974.

[17] J. Tits, Endliche Spiegelungsgruppen, die als Weylgruppen auftreten, Invent. Math. 43 (1977), 283 - 295.

[18] J. Tits, R. Weiss, Moufang Polygons, Springer Monographs in Mathematics, 2002.

[19] J. Truss, The group of the countable universal graph, Math. Proc. Camb. Phil. Soc. 98 (1985) 213-245.

[20] H. Van Maldeghem, Generalized polygons, Birkhäuser (1998)

Katrin Tent

Institut für Mathematische Logik

Fachbereich Mathematik

Universität Münste Einsteinstrasse 62

48149 Münster

Germany

Email: tent@uni-muenster.de 